and contemporary concepts of disease causation. It would seem from King's analysis that medicine has its own internal impetus for change, whereas most historians would place more emphasis on social and professional factors.

These lacunae in King's book by no means negate its value. It is a work which repays thoughtful reading, not least for the way it laments sloppy medical thinking in any age, our own included.

W.F. Bynum is at the Wellcome Institute for the History of Medicine, London, and a co-editor of the Dictionary of the History of Science (Macmillan/Princeton University Press, 1981).

\title{
Unity in diversity - or how to choose your animal
}

\section{R.B. Moreton}

Membrane Physiology of Invertebrates. Edited by Ronald B. Podesta. Pp.664. ISBN 0-8247-1503-9. (Dekker: 1982.) $\$ 87.50$.

IN THE animal kingdom, invertebrates not only outnumber the vertebrates to an unimaginable extent, but also present a fascinating diversity of form and function, combined with a surprising unity of basic mechanisms. To those of us who study a specific tissue in depth this provides an opportunity to pick those animals where the particular tissue is especially large, simple or well-developed. Thus, as every physiologist knows, the squid has a giant axon, the sea-urchin lays eggs, the crayfish has a stretch-receptor, and so on. This theme is developed by the late P.C. Caldwell in the introduction to this book, and it runs through the entire volume despite efforts by some authors to collect more general information: invertebrate physiology is just like that.

Membrane Physiology of Invertebrates is a collection of essays by specialist authors; arranged roughly in order of complexity of the organisms covered. It begins with an outline of our disappointingly sketchy understanding of how Amoeba feeds, and a detailed analysis of the electrophysiology of the "slipper microbe" Paramecium, whose genetic variations have been exploited to study particular membrane functions in isolation.

A chapter on coelenterates (jellyfish and hydroids) provides interesting examples of specialized intercellular junctions, and of the way in which development, for example the growth of new "heads", is controlled by cellular secretions. Intercellular junctions are still more important in the helminths, dealt with in the next contribution, where whole sheets of cells can be fused to form the body-wall. Annelids are covered in chapters on epithelial transport (osmoregulation, digestion and excretion) and excitble cells. The former presents a mainly qualitative study of "what happens where"; the latter begins with a rather confusing attempt to compare the properties of nerve cells in leech and earthworm with those of other groups, but gets into its stride with quantitative modelling of the giant axon of Myxicola. Crustacea are treated similariy, with a detailed and well-illustrated chapter on the gastrointestinal tract, and a good summary of the diverse types of nerve cells and sensory organs that have been studied, including a brief section on barnacle photoreceptors. A chapter by G.A. Gerencser on molluscan digestion presents mainly the author's own work, but refers to other reviews on osmoregulation and transport across the bodywall. The much larger pool of knowledge on molluscan nerve cells is covered, inevitably rather selectively, by F.E. Dudek, who concentrates on gastropods perhaps enough has been written about the squid axon for the time being! Dudek does include neuromodulators and neuropeptides, however, usefully integrating this relatively new topic with more conventional ideas on synaptic transmission.

Insects are represented by a contribution on gut physiology by W.R. Harvey, which includes an exposition of the thermodynamics of active transport systems, and by a neat summary of the properties of insect muscle, written by F.M. Ashcroft. There is no chapter on insect nerve cells, perhaps due to lack of space to treat this large subject properly. The book ends, unexpectedly, with a short chapter on the echinoderms, dealing mainly with the interesting electrical and chemical events accompanying fertilization of the egg.

This is not a book for the novice: most of the material is detailed and technical, with a fair amount of theory. Rather, it gives a comprehensive view "over the fence" for the specialist in transport or excitable tissue physiology, or for the researcher seeking to enter a new field. The title is misleading, in that much of the material relates to whole epithelia rather than membranes as such. But as a summary of our current knowledge of epithelial transport and excitable tissue physiology, right accross the invertebrate kingdom, the book is about as up-to-date as can be expected given the inevitable publication delays. Certainly, it should be in every library.

R.B. Moreton is a Principal Scientific Officer with the ARC Unit of Invertebrate Chemistry and Physiology, Department of Zoology, University of Cambridge.

\section{Molecular shapes}

\author{
M.G.B. Drew
}

Inorganic Stereochemistry. By David L. Kepert. Pp.227. ISBN 3-540-10716-9; 0-387-10716-9. (Springer-Verlag: 1982.) DM154, \$71.70.

CHEMISTs have always had a fundamental interest in the shapes of molecules and in evaluating the reasons for the adoption of a particular geometry. There are two theoretical approaches to such problems: the minimization of non-bonded repulsions and molecular orbital calculations. In this book, Professor Kepert uses simple repulsion theory to evaluate the various geometries of the metal coordination spheres of inorganic complexes in which one central metal atom is surrounded by 4-12 donor atoms.

The author takes each coordination number in turn and calculates the potential energy surface, allowing for possible combinations of unidentate and multidentate ligands. The theoretical results are then compared to the observed structural data provided by $\mathrm{X}$-ray analysis. The cnormous amount of structural information is thoroughly assimilated and well organized. There is excellent agreement between theory and experimental data, and in all the book amply demonstrates the validity of the repulsion approach to stereochemistry.

Kepert's work has undoubtedly been a major contribution to our understanding of molecular gcometry. However it does not seem to have had much impact on the way in which chemists look at molecular geometry. There are probably two main reasons for this. First, the parameters used for the potential energy curves are often not simple to calculate; structural chemists prefer to analyse geometry on the basis of bond lengths, bond angles and $\delta$ angles and find Kepert's parameters unnecessarily complicated. Secondly, he does not compare his results with those obtained by other methods. To take just one example, many people find that $\delta$ angles are useful structural parameters but they are not mentioned here. Nor indeed are the results of molecular orbital calculations.

It is this single-mindedness that is both the strength and weakness of the Kepert approach: whilst the book is selfcontained, it is not intended to provide a balanced view of current ideas on stereochemistry.

It is certainly useful to have Kepert's work brought together in one volume. However the book follows closely a series of reviews written for Progress in Inorganic Chemistry over the past few years; and those who have the previous articles may well find this present volume of limited additional value.

M.G.B. Drew is a Lecturer in Chemistry at the University of Reading. 